CARDIOVASCULAR MEDICINE

\title{
Survival trends among Danish patients undergoing coronary angiography for known or suspected ischaemic heart disease: a population based follow up study, 1992-2000
}

\author{
S P Johnsen, J Videbæk, L Pedersen, R Steffensen, R Videbæk, T Niemann, T T Nielsen, \\ H T Sørensen
}

Heart 2006;92:27-31. doi: 10.1136/hrt.2004.053314

\begin{abstract}
Objective: To determine, from population based clinical data, changes in the survival of Danish patients examined by coronary angiography for known or suspected ischaemic heart disease (IHD) during the 1990s.

Design: Follow up study.

Setting: The departments of cardiology at Rigshospitalet, Copenhagen University Hospital, and Skejby Hospital, Aarhus University Hospital, Denmark.

Patients: Patients with IHD ( $\mathrm{n}=7021$ ) who underwent first time coronary angiography in 1992,1996 , or 2000.

Main outcomes measures: Three year survival was compared between cohorts and with that of the general population. Cox proportional hazards regression was used to estimate mortality ratios adjusted for differences in patient characteristics.

Results: Survival improved substantially - for example, adjusted mortality ratio was 0.69 (95\% confidence interval (Cl) 0.55 to 0.87) when comparing patients from 2000 with patients from 1992. The absolute standardised survival rates after three years of follow up were $87.1 \%(95 \% \mathrm{Cl} 85.4 \%$ to $88.8 \%), 89.9 \%$ (95\% Cl $88.5 \%$ to $91.3 \%$ ), and $91.2 \%(95 \% \mathrm{Cl} 90.3 \%$ to $92.1 \%$ ) among patients examined in 1992,1996 , and 2000, respectively. The improvement was not explained by the improvement in overall survival in the general population during the study period.

Conclusions: The survival of Danish patients with known or suspected IHD appears to have improved substantially during the 1990s.
\end{abstract}

See end of article for authors' affiliations

Correspondence to: Dr Soren P Johnsen, Department of Clinical Epidemiology, Aarhus University Hospital and Aalborg Hospital, Ole Worms Allé 150, DK8000 Aarhus C, Denmark; spi@dce.au.dk

Accepted 17 March 2005 Published Online First 6 April 2005

M ortality from ischaemic heart disease (IHD) has been decreasing for at least two decades in Western Europe, the USA, and Australia. ${ }^{1-4}$ The decrease has been linked to primary and secondary prevention efforts, as well as to improvements in acute care. ${ }^{5-7}$ However, long term trends in mortality are hard to interpret, as effects of changes in incidence cannot be separated from changes in survival. Interpretation is further complicated by changes in diagnostic criteria for IHD and in the pattern of IHD presentation among hospitalised patients. Fewer patients now present with severe IHD, in particular myocardial infarction with extensive myocardial damage. ${ }^{8} 9$

Research on the prognosis of patients with IHD is most often based on clinical trials or administrative data, such as hospital discharge registries or insurance claims databases. ${ }^{10-16}$ Both approaches have important limitations. Patients in clinical trials may not be representative of the total patient population; they are typically recruited during a limited time span; and length of follow up tends to be relatively short. Routinely collected administrative data often lack clinical information that can ensure comparability of patient populations over time.

Relatively few studies have examined changes in the survival of patients with IHD in population based data supplemented by clinical information. ${ }^{14-18}$ Furthermore, few studies have compared trends in survival of patients with IHD with overall survival trends for the general population. ${ }^{10}$

Our study examined trends in survival rates in a population based sample of Danish patients with known or suspected IHD between 1992 and 2000, taking differences in patient characteristics into account and comparing their survival with survival in the general population.

\section{METHODS}

\section{Design and study population}

We conducted this follow up study among three cohorts of patients with known or suspected IHD $(\mathrm{n}=7025)$ : cohort $\mathrm{l}$ underwent first time coronary angiography in 1992; cohort 2 in 1996; and cohort 3 in 2000. The procedures took place at two major departments of cardiology (Rigshospitalet, Copenhagen University Hospital, and Skejby Hospital, Aarhus University Hospital). These hospitals provided invasive cardiac services for the population living in Copenhagen and the counties of Frederiksborg, Roskilde, Vestsjælland, Storestrøm, Bornholm, Aarhus, Viborg, and Ringkjøbing, with a total of 2.9 million inhabitants (about $55 \%$ of the total Danish population). Patients under 18 years were excluded $(\mathrm{n}=4)$. The Danish National Health Service provides tax supported health care for all citizens. It ensures free access to general practitioners, hospitals, and public clinics and refunds a variable proportion of the costs of prescribed drugs.

Abbreviations: $C A B G$, coronary artery bypass grafting; $\mathrm{Cl}$, confidence interval; EUROASPIRE, European action on secondary prevention through intervention to reduce events; ICD, International classification of diseases; IHD, ischaemic heart disease; PCl, percutaneous coronary intervention 


\section{Patient characteristics}

We obtained the following data from medical records or local clinical databases: age, sex, indication for coronary angiography-that is, acute or semi-acute (patient unstable or waiting time $\leqslant 7$ days)-and angiographic status (no significant stenosis, single vessel disease, double vessel disease, triple vessel disease, or proximal left main coronary artery stenosis). "No significant stenosis" was defined as stenosis occupying less than $50 \%$ of the lumen. All data were recorded on standardised forms at the time of coronary angiography and were available as a hard copy or electronically, depending on the year of examination.

Data on cardiovascular and non-cardiovascular co-morbidities at baseline were obtained from the Danish National Registry of Patients. This registry, established in 1977, contains records on $99.4 \%$ of discharges from non-psychiatric hospitals in Denmark. ${ }^{19}$ The data include civil registry number, dates of admission and discharge, surgical procedures performed, and up to 20 discharge diagnoses coded by medical doctors at discharge until 1993 according to the Danish version of the International classification of diseases, 8th revision (ICD-8) and subsequently according to the national version of ICD-10. ${ }^{19}$ Data from the National Registry of Patients were used to calculate a co-morbidity index score for each patient as described by Charlson et al. ${ }^{20}$ The Charlson index covers 19 major disease categories, including acute myocardial infarction, congestive heart failure, cerebrovascular disease, and cancer, weighted according to their prognostic impact on patient survival. The index was previously adapted for use with hospital discharge registry data. ${ }^{21}$

We defined three levels of co-morbidity for each patient based on a complete hospital discharge history from all nonpsychiatric Danish hospitals for the 10 year period preceding the index date, as follows: 0 co-morbidities (none) for patients with no recorded underlying diseases included in the Charlson index; 1-2 co-morbidities (low); and $>2$ comorbidities (high).

Information on coronary revascularisation proceduresthat is, percutaneous coronary intervention (PCI) or coronary artery bypass grafting (CABG) - performed in direct connection with the baseline coronary angiography or within one year of follow up was also obtained from the National Registry of Patients.

\section{Data on survival}

Records in population based registries can be accurately linked in Denmark through the civil registry number-a unique, permanent personal identification number given to every Danish citizen. We obtained information on survival through record linkage with the Civil Registration System, which has electronic records on all changes in vital status, including change of address, date of emigration, and date of death for the entire Danish population since 1968.

To compare patient survival with survival among the general population, we selected a representative sample of the general population for each of the three patient cohorts. Ten people from the general population were selected for each patient, frequency matched on year of birth and sex.

\section{Statistical analysis}

Characteristics of the three cohorts were compared by linear regression for the continuous variables (such as age) and $\chi^{2}$ tests for the categorical variables.

Follow up began on the date of the first coronary angiography and ended on the date of death, the date of emigration, or after three years, whichever came first.

We used Cox proportional hazards regression to estimate mortality ratios adjusted for prognostic factors-that is, age, sex, indication for coronary angiography, angiographic status, and level of co-morbidity. The 1992 cohort served as the reference for all analyses.

We also carried out analyses with separate Cox models, stratifying by age, sex, indication for angiography, angiographic status, level of co-morbidity, and revascularisation treatment group. We used the likelihood ratio test to evaluate homogeneity.

To take into account any changes in the overall survival of the general population during the study period, we compared the survival of the three samples from the general population after having standardised the 1992 and 1996 samples to the age and sex distribution of the 2000 sample. We then adjusted for the change in overall survival in the comparisons between the three patient cohorts in the Cox models.

Lastly, survival curves for the three cohorts were plotted to compare the absolute survival rates after having standardised the 1992 and 1996 cohorts to the age, sex, indication for angiography, angiographic status, and co-morbidity distribution of the 2000 cohort. We compared the survival curves by log rank tests.

The assumption of proportional hazards in the Cox models was evaluated by graphic assessment. There were indications that the assumption was not fulfilled for the entire follow up period and some caution is therefore warranted when interpreting the risk estimates. We estimated $95 \%$ confidence intervals (CIs) for all analyses. Our statistical analyses were done with SAS version 8.00 (SAS Institute Inc, Cary, North Carolina, USA).

\section{RESULTS}

Table 1 shows demographic and medical characteristics of each of the three cohorts, totalling 7021 patients. The number of patients undergoing first time coronary angiography increased by a factor of three between 1992 and 2000. Observed trends were a higher mean age, higher proportion of women, more patients with acute indications, more patients with no significant stenoses or single vessel disease, more patients undergoing a revascularisation procedure in direct connection with the baseline coronary angiography or within one year of follow up, and more patients with high comorbidity index scores $(\mathrm{p}<0.001)$. Furthermore, the median time from coronary angiography to revascularisation dropped dramatically as more patients were revascularised in direct connection with the baseline coronary angiography.

Combining all three cohorts, we identified a total of 792 deaths during follow up. This corresponds to a mortality of 40.6 deaths/1000 person years of risk.

Table 2 provides crude and adjusted mortality ratios during three years of follow up according to the year of first time coronary angiography for known or suspected IHD. No clear changes in mortality over time were seen in the crude analyses. However, after adjusting for covariates, we found a substantial improvement in survival, for example, the adjusted mortality ratio was 0.69 (95\% CI 0.55 to 0.87 ) when comparing patients from 2000 with patients from 1992. Age and angiographic status were the most influential confounding factors.

To evaluate the possibility that improved survival over time among patients with known or suspected IHD was restricted to certain subgroups of patients, we stratified our analyses according to age, sex, angiographic status, level of comorbidity, and revascularisation treatment group (table 3). Indications of improved survival over time were observed in all strata; however, not all mortality ratios reached significance. Although the mortality ratios varied, there were no significant differences in the stratified analyses.

The improvement in survival of patients with known or suspected IHD during the study period appeared not to be 
Table 1 Characteristics of patients examined by coronary angiography for known or suspected ischaemic heart disease in 1992, 1996, and 2000

\begin{tabular}{|c|c|c|c|}
\hline Patient characteristic & 1992 & 1996 & 2000 \\
\hline Number & 1578 & 1709 & 3734 \\
\hline \multicolumn{4}{|l|}{ Age (years)* } \\
\hline $19-49$ & $21.8 \%$ & $18.0 \%$ & $15.8 \%$ \\
\hline $50-69$ & $67.8 \%$ & $63.5 \%$ & $55.7 \%$ \\
\hline$\geqslant 70$ & $10.4 \%$ & $18.5 \%$ & $28.6 \%$ \\
\hline \multicolumn{4}{|l|}{ Sex* } \\
\hline Men & $73.3 \%$ & $71.3 \%$ & $65.6 \%$ \\
\hline Women & $26.7 \%$ & $28.7 \%$ & $34.4 \%$ \\
\hline \multicolumn{4}{|l|}{ Indication* } \\
\hline Acute & $5.8 \%$ & $24.3 \%$ & $44.0 \%$ \\
\hline Elective & $94.2 \%$ & $75.7 \%$ & $56.0 \%$ \\
\hline \multicolumn{4}{|l|}{ Angiographic status* } \\
\hline No significant stenoses & $14.6 \%$ & $20.2 \%$ & $27.1 \%$ \\
\hline 1 VD & $16.4 \%$ & $26.1 \%$ & $27.5 \%$ \\
\hline $2 \mathrm{VD}$ & $13.1 \%$ & $22.4 \%$ & $17.5 \%$ \\
\hline $3 \mathrm{VD}$ & $24.4 \%$ & $26.8 \%$ & $20.0 \%$ \\
\hline PLM stenosis & $4.3 \%$ & $4.5 \%$ & $6.3 \%$ \\
\hline Missing data & $27.3 \%$ & $0.0 \%$ & $1.5 \%$ \\
\hline \multicolumn{4}{|l|}{ Charlson co-morbidity index* } \\
\hline 0 & $34.1 \%$ & $35.7 \%$ & $35.9 \%$ \\
\hline $1-2$ & $58.9 \%$ & $54.4 \%$ & $51.3 \%$ \\
\hline$>2$ & $6.9 \%$ & $9.9 \%$ & $12.7 \%$ \\
\hline $\begin{array}{l}\text { Revascularisation at baseline or within } \\
\text { one year of follow up* }\end{array}$ & $47.5 \%$ & $54.7 \%$ & $58.8 \%$ \\
\hline Time to revascularisation (days) ${ }^{*} \dagger$ & $91(0-365)$ & $33(0-365)$ & $0(0-365)$ \\
\hline Total follow up (years) & 4357 & 4737 & 10408 \\
\hline Number of deaths during follow up & 188 & 185 & 419 \\
\hline
\end{tabular}

explained by the improvement in overall survival for the general population during the same period. Thus, the adjusted mortality ratios for the 1996 and 2000 cohorts were 0.88 ( $95 \%$ CI 0.74 to 1.05 ) and 0.77 (95\% CI 0.63 to 0.95 ), respectively, when we further adjusted for the improvement in overall survival of the general population during the study period.

Figure 1 presents standardised survival functions for patients in the 1992, 1996, and 2000 cohorts. The absolute standardised survival rates after three years of follow up were $87.1 \%$ ( $95 \%$ CI 85.4 to 88.8 ), $89.9 \%$ (95\% CI 88.5 to 91.3 ), and $91.2 \%$ (95\% CI 90.3 to 92.1 ) for the 1992, 1996, and 2000 cohorts, respectively.

\section{DISCUSSION}

We found a major improvement in the survival of Danish patients with known or suspected IHD during the 1990s in this population based follow up study. The improvement was found consistently in all subgroups of patients examined, although the improvement did not reach significance in all subgroups. The improvement could not be explained by changes during the study period in the available patient

Table 2 Crude and adjusted mortality ratios (MRs) during three years of follow up among patients examined with first time coronary angiography for known or suspected ischaemic heart disease in 1992, 1996, and 2000

\begin{tabular}{llll}
\hline & $\begin{array}{l}\text { Deaths } \\
\text { during } \\
\text { follow up }\end{array}$ & $\begin{array}{l}\text { Crude MR } \\
(95 \% \mathrm{Cl})\end{array}$ & Adjusted MR* $(95 \% \mathrm{Cl})$ \\
\hline 19921578 & 188 & 1.0 (reference) & 1.0 (reference) \\
19961709 & 185 & $0.91(0.74$ to 1.11$)$ & 0.83 (0.66 to 1.06$)$ \\
20003734 & 419 & $0.94(0.80$ to 1.11$)$ & $0.69(0.55$ to 0.87$)$ \\
\hline
\end{tabular}

*Adjusted for age, sex, indication (acute or elective), angiographic status, and Charlson co-morbidity index.

$\mathrm{Cl}$, confidence interval. characteristics including age, sex, indication (acute or elective), angiographic status, and Charlson co-morbidity index. Furthermore, the improvement in survival of patients with IHD appeared not to be explained by the improvement in overall survival of the general population during the same period.

The strength of our study is a prospective population based design with complete long term follow up, minimising the risk of selection bias. The availability of detailed individual level clinical data, including angiographic status, level of comorbidity, and revascularisation during follow up, made it possible to adjust for changes in patient characteristics during the study period. Furthermore, the study included a direct comparison of the improvements in survival between patients with IHD and the general population during the period of observation. This comparison is important, since survival among the general population may have changed because of improvements in the prevention and treatment of diseases other than IHD. These improvements also apply to patients with IHD, who often have coexisting diseases that may eventually cause death. Not taking this into consideration would have led us to overestimate improvements in survival solely related to IHD.

The study was limited by a lack of postangiographic data, including changes in lifestyle factors such as smoking, physical inactivity, and diet, as well as use of cardiovascular drugs. This prevented us from isolating the reasons for improved survival. Furthermore, although we adjusted for a number of differences in patient characteristics, our findings still could have been influenced by undetected differences due to missing data, such as ejection fraction, or due to the use of relatively crude data for some variables, such as level of co-morbidity. However, it should be noted that the validity of some hospital discharge diagnoses, such as acute myocardial infarction, appears to be quite high in the Danish National Registry of Patients. ${ }^{22}$

Our study accords with and extends the findings of a number of other studies on western populations. Together, 
Table 3 Crude and adjusted MRs stratified by age, sex, angiographic status, level of co-morbidity, and revascularisation treatment group among patients examined with first time coronary angiography for known or suspected ischaemic heart disease in 2000 (1992 is used as reference)

\begin{tabular}{|c|c|c|c|c|}
\hline & \multicolumn{2}{|l|}{1996 v 1992} & \multicolumn{2}{|l|}{$2000 \vee 1992$} \\
\hline & Crude MR $(95 \% \mathrm{CI})$ & Adjusted $\mathrm{MR}^{*}(95 \% \mathrm{Cl})$ & Crude MR (95\%) & Adjusted $\mathrm{MR}^{*}(95 \% \mathrm{CI})$ \\
\hline \multicolumn{5}{|l|}{ Age (years) } \\
\hline $19-49$ & $0.47(0.22$ to 1.02$)$ & $0.51(0.22$ to 1.18$)$ & $0.58(0.31$ to 1.05$)$ & $0.51(0.22$ to 1.18$)$ \\
\hline $50-69$ & $0.82(0.63$ to 1.06$)$ & $0.89(0.66$ to 1.19$)$ & $0.65(0.52$ to 0.82$)$ & $0.67(0.51$ to 0.89$)$ \\
\hline$\geqslant 70$ & 0.91 (0.62 to 1.34$)$ & 0.91 (0.54 to 1.54 ) & $0.88(0.63$ to 1.23$)$ & $0.82(0.49$ to 1.35$)$ \\
\hline \multicolumn{5}{|l|}{ Sex } \\
\hline Men & $0.94(0.74$ to 1.18$)$ & $0.88(0.67$ to 1.15$)$ & $0.92(0.75$ to 1.13$)$ & $0.70(0.54$ to 0.90$)$ \\
\hline Women & $0.84(0.56$ to 1.26$)$ & $0.74(0.45$ to 1.21$)$ & $0.99(0.71$ to 1.39$)$ & 0.69 (0.44 to 1.08$)$ \\
\hline \multicolumn{5}{|l|}{ Indication } \\
\hline Acute & $0.90(0.50$ to 1.62$)$ & $0.81(0.45$ to 1.46$)$ & $0.94(0.55$ to 1.62$)$ & $0.73(0.42$ to 1.26$)$ \\
\hline Elective & $0.84(0.67$ to 1.05$)$ & $0.85(0.65$ to 1.11$)$ & $0.72(0.59$ to 0.89$)$ & $0.66(0.51$ to 0.86$)$ \\
\hline \multicolumn{5}{|l|}{ Angiographic status } \\
\hline No significant stenoses & 0.89 (0.42 to 1.89$)$ & 0.72 (0.34 to 1.54$)$ & $1.16(0.62$ to 2.15$)$ & $0.69(0.36$ to 1.34$)$ \\
\hline 1 or 2 VD & $1.20(0.78$ to 1.83$)$ & $0.94(0.61$ to 1.45$)$ & $1.53(1.04$ to 2.24$)$ & $0.87(0.58$ to 1.31$)$ \\
\hline 3 VD or PLM stenosis & $1.25(0.93$ to 1.69$)$ & $0.84(0.62$ to 1.15$)$ & $1.21(0.92$ to 1.54$)$ & $0.63(0.46$ to 0.83$)$ \\
\hline \multicolumn{5}{|l|}{ Charlson co-morbidity index } \\
\hline 0 & $0.66(0.42$ to 1.03$)$ & $0.72(0.43$ to 1.20$)$ & $0.61(0.42$ to 0.89$)$ & $0.61(0.38$ to 0.99$)$ \\
\hline $1-2$ & $0.97(0.74$ to 1.28$)$ & $0.92(0.67$ to 1.27$)$ & $0.95(0.75$ to 1.20$)$ & $0.70(0.51$ to 0.96$)$ \\
\hline$>2$ & $0.83(0.54$ to 1.27$)$ & $0.74(0.45$ to 1.20$)$ & $0.86(0.60$ to 1.24$)$ & $0.68(0.43$ to 1.07$)$ \\
\hline \multicolumn{5}{|c|}{ Revascularisation at baseline or within one year of follow up } \\
\hline Yes & $0.98(0.72$ to 1.32$)$ & $0.85(0.60$ to 1.20$)$ & $0.90(0.70$ to 1.18$)$ & 0.61 (0.44 to 0.84$)$ \\
\hline No & $0.79(0.60$ to 1.05$)$ & $0.88(0.63$ to 1.24$)$ & $0.92(0.73$ to 1.16$)$ & $0.87(0.64$ to 1.19$)$ \\
\hline
\end{tabular}

*Adjusted for age, sex (except when stratified by sex), indication (acute or elective) (except when stratified by indication), angiographic status (except when stratified by angiographic status), and Charlson co-morbidity index (except when stratified by Charlson co-morbidity index).

they show an encouraging trend in the survival of patients with a hospital discharge diagnosis of IHD during the past two decades. ${ }^{2-4} 7^{10-18}$ The majority of existing studies have focused on patients with acute myocardial infarction, whereas data on survival trends in patients with stable angina are sparse. Little attention has been paid to the changing pattern of IHD, characterised by relatively more patients with milder forms of IHD. However, the consistency with which the survival improvement has been found in various settings and patient populations indicates that the findings most likely reflect a true and substantial improvement in both short and relative long term survival of patients with IHD in most Western populations.
Determinants of illness outcome should be taken into account $^{23}$ when considering possible explanations for improved survival. These include patient characteristics and the illness itself, diagnostic tests used to identify the cause and severity of the disease, treatments, quality of care, and level of patient compliance. ${ }^{23}$ In our study, we controlled for major patient characteristics, such as severity of IHD, and for choice of diagnostic tests as possible explanations for the improved survival; however, treatment, quality of care, and patient compliance may have changed.

Increased use of effective treatments, including aspirin and other antiplatelet drugs, $\beta$ blockers, angiotensin converting enzyme inhibitors, statins, and coronary revascularisation,

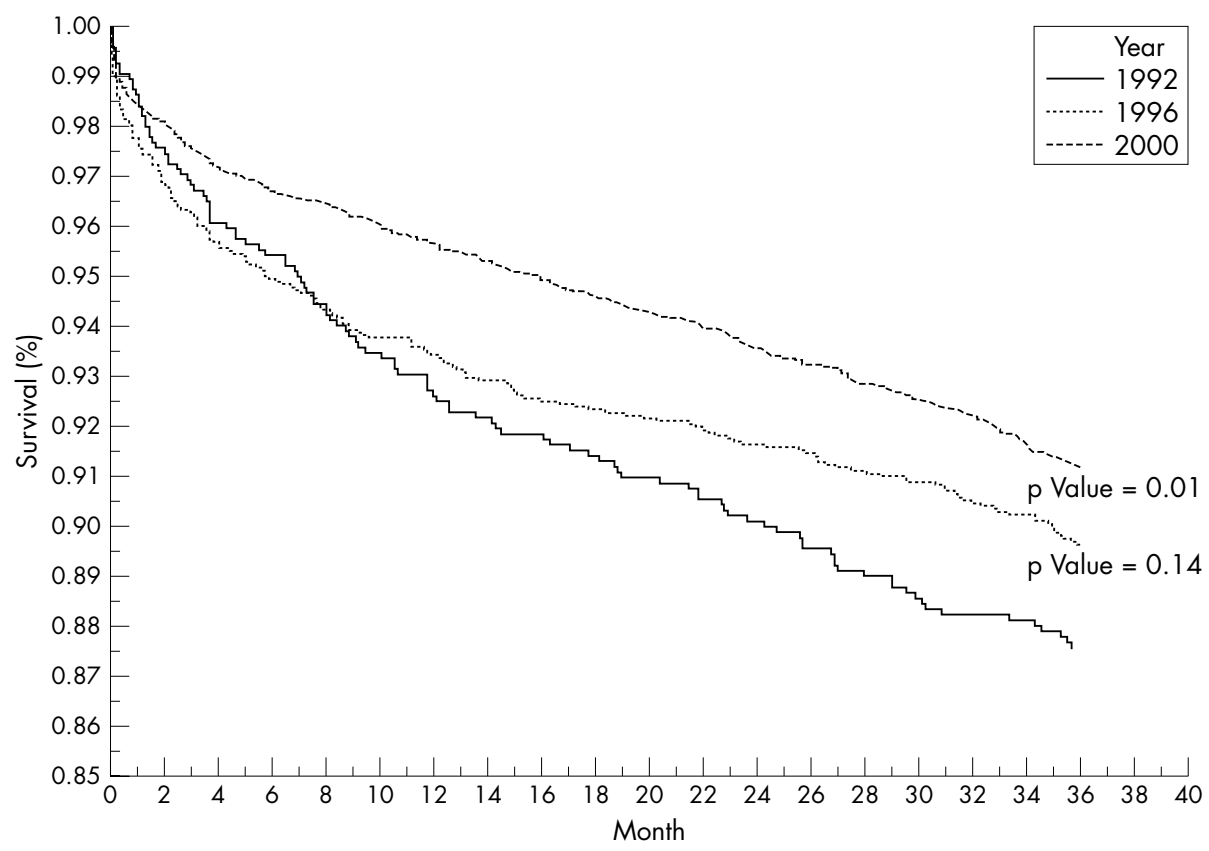

Figure 1 Survival functions among patients examined by first time coronary angiography for known or suspected ischaemic heart disease in 1992,1996 , and 2000 standardised to patient characteristics during 2000 lage, sex, indication for angiography, and angiographic status). Survival is compared by log rank tests with the patients from 1992 as reference. Please note that the $y$ axis does not start at zero. 
have been previously identified as the primary reason for improved short term survival of patients with acute myocardial infarction. ${ }^{74}$ The improvement found in our study paralleled the implementation of a national plan in Denmark in 1993 aimed at rapidly expanding the capacity for performing interventional cardiological procedures, including coronary angiography, PCI, and CABG. As a consequence of this initiative, the total number of coronary angiograms and of PCI or CABG performed in Denmark increased by $387 \%$ and $331 \%$, respectively, during the 1992-2000 period. However, the proportion of patients undergoing revascularisation procedures during follow up did not change dramatically across the 1992, 1996, and 2000 patient cohorts. Thus, the increased capacity for interventional procedures in itself is not likely to explain the improvement in survival. At the same time, improvements in the effectiveness of the procedures, such as increased use of coronary stents during PCI procedures for preventing restenosis and in particular implementation of potent antithrombotic regimens, are likely to have been important. This is reinforced by our findings of a substantial improvement during the 1990s in survival among patients undergoing revascularisation during follow up.

Furthermore, use of cardiovascular drugs for secondary prevention appears to have increased among Danish patients with IHD during the 1990s. ${ }^{25}{ }^{26}$ However, as shown in the EUROASPIRE (European action on secondary prevention through intervention to reduce events) I and II surveys, many patients with IHD still do not reach the recommended treatment goals and the proportion of patients who are either smokers or obese appears to be stable or even increasing. ${ }^{27}$ Moreover, the improvement in survival may not apply to all categories of patients with IHD. Long term survival after out of hospital cardiac arrest remains poor, ${ }^{28}$ and about $70 \%$ of all deaths caused by IHD occur unexpectedly and outside the hospital, often among people with undiagnosed IHD. ${ }^{28-30}$ Thus, there is clear potential for further improving the survival of patients with IHD in western populations.

In conclusion, we found a major improvement in the survival of a population based sample of Danish patients evaluated by coronary angiography for known or suspected IHD during the 1990s. The improvement appeared not to be explained by differences in available patient characteristics, including age, sex, indication (acute or elective), angiographic status, and Charlson co-morbidity index, or by the improvement in overall survival in the general population during the same period. Several factors, including a substantial increase in the use of coronary revascularisation and cardiovascular drugs for secondary prevention, may underlie the improvement.

\section{Authors' affiliations \\ S P Johnsen, L Pedersen, H T Sørensen, Department of Clinical Epidemiology, Aarhus University Hospital, Aarhus, Denmark J Videbæk, The Danish Heart Foundation, Copenhagen, Denmark R Steffensen, Department of Cardiology, Hillerød Hospital, Hillerød, Denmark \\ R Videbæk, The Heart Centre, Rigshospitalet, Copenhagen University Hospital, Copenhagen, Denmark \\ T Niemann, Department of Medicine, Herning Hospital, Herning, Denmark \\ T T Nielsen, Department of Cardiology, Skejby Hospital, Aarhus University Hospital, Aarhus, Denmark \\ Source of funding: Danish Heart Foundation}

The study was approved by The Danish Data Protection Agency (J nr 2001-41-0903).

\section{REFERENCES}

1 Beaglehole R. International trends in coronary heart disease mortality, morbidity, and risk factors. Epidemiol Rev 1990;12:1-15.

2 Levi F, Lucchini F, Negri E, et al. Trends in mortality from cardiovascular and cerebrovascular diseases in Europe and other areas of the world. Heart 2002;88:119-24.

3 Rosamond WD, Chambless LE, Folsom AR, et al. Trends in the incidence of myocardial infarction and in mortality due to coronary heart disease, 1987 to 1994. N Engl J Med 1998;339:861-7.

4 Abildstrom SZ, Rasmussen S, Rosén $M$, et al. Trends in incidence and case fatality rates of acute myocardial infarction in Denmark and Sweden. Heart 2003:89:507-11.

5 Kuulasmaa K, Tunstall-Pedoe H, Dobson A, et al. Estimation of contribution of changes in classic risk factors to trends in coronary-event rates across the WHO MONICA project populations. Lancet 2000;355:675-87.

6 Tunstall-Pedoe $\mathbf{H}$, Vanuzzo D, Hobbs $M$, et al. Estimation of contribution of changes in coronary care to improving survival, event rates, and coronary heart disease mortality across the WHO MONICA project populations. Lancet 2000;355:688-700

7 Heidenreich PA, McClellan M. Trends in treatment and outcomes for acute myocardial infarction: 1975-1995. Am J Med 2001:110:165-74.

8 Luepker RV, Apple FS, Christenson RH, et al. Case definitions for acute coronary heart disease in epidemiology and clinical research studies. Circulation 2003; 108:2543-9

9 Murphy NF, Maclntyre K, Capewell S, et al. Hospital discharge rates for suspected acute coronary syndromes between 1990 and 2000: population based analysis. BMJ 2004;328:1413-4.

10 Bronnum-Hansen $\mathbf{H}$, Jorgensen T, Davidsen $M$, et al. Survival and cause of death after myocardial infarction: the Danish MONICA study. J Clin Epidemiol 2001;54:1244-50.

11 Abildstrom SZ, Rasmussen S, Madsen M. Significant decline in case fatality after acute myocardial infarction in Denmark: a population based study from 1994 to 2001. Scand Cardiovasc J 2002;36:287-91.

12 Naylor CD, Chen E. Population-wide mortality trends among patients hospitalized with acute myocardial infarction: the Ontario experience, 19811991. J Am Coll Cardiol 1994;24:1431-8.

13 Gottlieb S, Goldbourt U, Boyko V. Improved outcome of elderly patients (>or $=75$ years of age) with acute myocardial infarction from 1981-1983 to 1992-1994 in Israel. The SPRINT and thrombolytic survey groups. Secondary prevention reinfarction israel nifedipine trial. Circulation 1997;95:342-50.

14 Tu JV, Naylor CD, Austin P. Temporal changes in the outcomes of acute myocardial infarction in Ontario, 1992-1996. Can Med Assoc J 1999;161:1257-61

15 Herlitz J, Dellborg M, Karlson BW, et al. Prognosis after acute myocardial infarction continues to improve in the reperfusion era in the community of Göteborg. Am Heart J 2002; 144:89-94.

16 Quan H, Cujec B, Jin Y, et al. Acute myocardial infarction in Alberta: temporal changes in outcomes, 1994 to 1999. Can J Cardiol 2004;20:213-9.

17 Fox CS, Evans JC, Larson MG, et al. Temporal trends in coronary heart disease mortality and sudden cardiac death from 1950 to 1999. Circulation 2004; 1 10:522-7.

18 Ergin A, Muntner P, Sherwin R, et al. Secular trends in cardiovascular disease mortality, incidence, and case fatality rates in adults in the United States. Am J Med 2004;117:219-27.

19 Andersen TF, Madsen M, Jorgensen J, et al. The Danish national hospital register: a valuable source of data for modern health sciences. Dan Med Bull 1999;46:263-8.

20 Charlson ME, Pompei P, Ales KL, et al. A new method of classifying prognostic comorbidity in longitudinal studies: development and validation. J Chronic Dis 1987;40:373-83.

21 Deyo RA, Cherkin DC, Ciol MA. Adapting a clinical comorbidity index for use with ICD-9-CM administrative databases. J Clin Epidemiol 1992;45:613-9.

22 Madsen M, Davidsen M, Rasmussen S, et al. The validity of the diagnosis of acute myocardial infarction in routine statistics: a comparison of mortality and hospital discharge data with the Danish MONICA registry. J Clin Epidemiol 2003;56: 124-30.

23 Sackett DL, Haynes RB, Guyatt GH, et al. Clinical epidemiology. A basic science for clinical medicine, 2nd edn. Boston: Little Brown, 1991:305-33.

24 Stenestrand $U$, Wallentin L. Early statin treatment following acute myocardial infarction and 1-year survival. JAMA 2001;285:430-6.

25 Videbæk J, Madsen M. Heart statistics. Copenhagen: The Danish Heart Foundation and National Institute of Public Health, 2001.

26 Riahi S, Fonager K, Toft E, et al. Use of lipid-lowering drugs during 1991-98 in Northern Jutland, Denmark. Br J Clin Pharmacol 2001;52:307-11.

27 Anon. EUROASPIRE I and II Group. Clinically reality of coronary prevention guidelines: a comparison of EUROASPIRE I and II in nine countries, Lancet 2001;357:995-1001.

28 Engdahl J, Bång A, Lindqvist J, et al. Time trends in long-term mortality after out-of-hospital cardiac arrest, 1980 to 1998, and predictors for death. Am J Heart J 2003;145:826-33.

29 Tunstall-Pedoe H, Morrison C, Woodward M, et al. Sex differences in myocardial infarction and coronary deaths in the Scottish MONICA population of Glasgow 1985 to 1991: presentation, diagnosis, treatment, and 28-day case fatality of 3991 events in men and 1551 events in women. Circulation 1996;93:1981-92.

30 Norris RM. Fatality outside hospital in acute coronary events in three British health districts, 1994-5. United Kingdom heart attack study collaborative group. BMJ 1998;316:1065-70. 